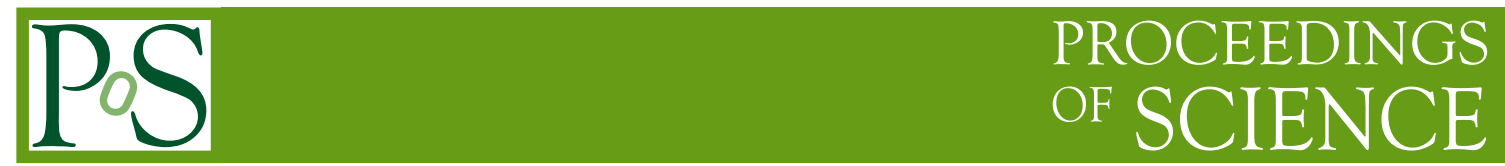

\title{
New results using the razor at the LHC
}

\author{
William Reece* \\ On behalf of the CMS Collaboration \\ Physics Department, CERN, Switzerland \\ E-mail: Will.Reecedcern.ch
}

\begin{abstract}
The razor variables provide a sensitive and model independent way to search for the pairproduction of massive new particles at the LHC. A number of different analyses have been performed by the CMS collaboration using these variables, resulting in stringent limits for many classes of new physics models. In this note, the status of these searches is presented using data taken by the CMS detector during the $2011 \mathrm{LHC}$ proton-proton run at $7 \mathrm{TeV}$.
\end{abstract}

36th International Conference on High Energy Physics,

July 4-11, 2012

Melbourne, Australia

* Speaker. 


\section{Introduction}

The CMS detector is a general purpose high-energy physics experiment in the large hadron collider (LHC) at CERN [1]. During the 2011 proton-proton run of the LHC, the detector recorded $4.98 \pm 0.11 \mathrm{fb}^{-1}$ of data passing basic quality requirements at a centre of mass energy of $7 \mathrm{TeV}$. In this note, searches by the CMS collaboration for the decay of massive new particles using the razor variables are presented. No significant excess is seen, so stringent limits are placed on relevant masses and cross-sections of new particles at the LHC. These results are interpreted within the framework of supersymmetry.

\section{The Razor Variables}

The razor kinematics are based on the generic process of the pair production of two heavy particles, each decaying to an unseen particle plus jets [2]. This includes supersymmetric signals with complicated and varied decay chains, or the simplest case of a pair of squarks each decaying to a quark and an LSP. All such processes are treated on an equal footing by forcing every event into a dijet topology; this is done by combining all jets in the event into two megajets [3]. To the extent that the pair of megajets accurately reconstruct the visible portion of the underlying parent particle decays, the signal kinematics is equivalent to pair production of heavy squarks $\tilde{q}_{1}, \tilde{q}_{2}$, with $\tilde{q}_{i} \rightarrow \mathrm{j}_{i} \tilde{\chi}_{i}$, where the $\tilde{\chi}_{i}$ are LSPs and $\mathrm{j}_{i}$ denotes the visible products of the decays. For simplicity we will use the approximation that the $\mathrm{j}_{i}$ are massless.

In the $\tilde{q}_{1} \tilde{q}_{2}$ centre of mass $(\mathrm{CM})$ frame the energies of the visible decay products can be written

$$
\begin{aligned}
& E_{\mathrm{j}_{1}}=\frac{\gamma_{C M} M_{\Delta}}{2}\left(1+\beta_{C M} \hat{u}_{\tilde{q}} \cdot \hat{u}_{1}\right), \\
& E_{\mathrm{j}_{2}}=\frac{\gamma_{C M} M_{\Delta}}{2}\left(1+\beta_{C M} \hat{u}_{\tilde{q}} \cdot \hat{u}_{2}\right),
\end{aligned}
$$

where $\hat{u}_{\tilde{q}}$ is a unit vector in the direction of the first squark, $\hat{u}_{i}$ are unit vectors in the directions of the visible decay products, $\beta_{C M}$ is the boost parameter from the $\mathrm{CM}$ frame to the $\tilde{q}_{1}$ rest frame, and

$$
M_{\Delta} \equiv \frac{M_{\tilde{q}}^{2}-M_{\tilde{\chi}}^{2}}{M_{\tilde{q}}} .
$$

The energy distribution for the visible decay products as measured in the $\mathrm{CM}$ frame then peaks around $\left(\gamma_{C M} M_{\Delta}\right) / 2$. The strategy of the razor analysis is to approximate this frame, which is inaccessible due to the presence of two invisible particles in the final state, with a razor frame that is defined unambiguously from measured quantities in the lab frame. Event by event, razor frame observables then estimate the scale $M_{\Delta}$ seen above.

A razor frame is defined by finding a longitudinal boost from the lab frame to a frame where the visible energies can be written in terms of an overall scale that is manifestly invariant under longitudinal boosts. This then defines a razor frame where the scale of the visible energies is set by a quantity that should approximate $\gamma_{C M} M_{\Delta}$ in the (unknown) $\mathrm{CM}$ frame. The longitudinal boost used here is defined as:

$$
\beta_{L}^{R} \equiv \frac{p_{z}^{\mathrm{j}_{1}}+p_{z}^{\mathrm{j}_{2}}}{E_{\mathrm{j}_{1}}+E_{\mathrm{j}_{2}}}
$$


The razor boost $\beta_{L}^{R}$ defines a frame where the visible four-momenta reduce to

$$
\begin{aligned}
& p_{\mathrm{j}_{1}}=\left(\frac{1}{2}\left(M_{R}-\frac{\left(\vec{p}_{T}^{\mathrm{j}_{1}}-\vec{p}_{T}^{\mathrm{j}_{2}}\right) \cdot \vec{E}_{T}^{\text {miss }}}{M_{R}}\right), p_{T}^{\mathrm{j}_{1}}, p_{z}\right), \\
& p_{\mathrm{j}_{2}}=\left(\frac{1}{2}\left(M_{R}+\frac{\left(\vec{p}_{T}^{\mathrm{j}_{1}}-\vec{p}_{T}^{\mathrm{j}_{2}}\right) \cdot \vec{E}_{T}^{m i s s}}{M_{R}}\right), p_{T}^{\mathrm{j}_{2}},-p_{z}\right),
\end{aligned}
$$

where $M_{R}$ is the longitudinal boost invariant

$$
M_{R} \equiv \sqrt{\left(E_{\mathrm{j}_{1}}+E_{\mathrm{j}_{2}}\right)^{2}-\left(p_{z}^{\mathrm{j}_{1}}+p_{z}^{\mathrm{j}_{2}}\right)^{2}}
$$

and the longitudinal momentum $p_{z}$ is determined from the massless on-shell conditions. Here $M_{R}$ as defined by (2.7) is an estimator of $\gamma_{C M} M_{\Delta}$.

The next step of the razor strategy is to define a transverse observable that can also serve as an event-by-event estimator of the underlying scale $M_{\Delta}$. As usual for transverse quantities we expect $M_{\Delta}$ to be related to a kinematic edge rather than a peak. Especially useful is $M_{T}^{R}$, a kind of average transverse mass whose maximum value for signal events is $M_{\Delta}$ :

$$
M_{T}^{R} \equiv \sqrt{\frac{E_{T}^{m i s s}\left(p_{T}^{j 1}+p_{T}^{j 2}\right)-\vec{E}_{T}^{m i s s} \cdot\left(\vec{p}_{T}^{j 1}+\vec{p}_{T}^{j 2}\right)}{2}} .
$$

Given a global estimator $M_{R}$ and a transverse estimator $M_{T}^{R}$, the razor dimensionless ratio is defined as

$$
R \equiv \frac{M_{T}^{R}}{M_{R}}
$$

Signal events are characterized by the heavy scale $M_{\Delta}$, while backgrounds are not. Qualitatively we expect $M_{R}$ to peak for the signal over a steeply falling background. Thus the search for an excess of signal events in a tail of a distribution is recast as a search for a peak on top of a steeply falling standard model (SM) distribution.

\section{The Razor Inclusive Analysis}

The razor inclusive analysis [4] is constructed to be as model independent and inclusive as possible, while still giving sensitivity to a wide range of new physics models with decays that can be described in terms of the razor kinematics. Events are selected inclusively in the CMS high-level trigger (HLT) using the razor variables and then categorised into six final-states, known as boxes, as shown in Fig. 1. An unbinned maximum likelihood fit is used to extract the shape of the SM distribution, box-by-box, in a region dominated by background (the fit region). The background distribution in each box is then extrapolated out into a signal region, and the predicted yields are compared to those observed. An example fit is shown in Fig. 2 for the hadronic final state. No discrepancy between the data and the extrapolated background shape is seen, and so limits are set in a variety of models, including the constrained minimal supersymmetric standard model (CMSSM). To increase the signal efficiency and model independence of the analysis, the individual limits from the six final-state boxes are combined. The observed and expected limits in the CMSSM, as well as individual hadronic and combined leptonic limits are shown in Fig. 3. 


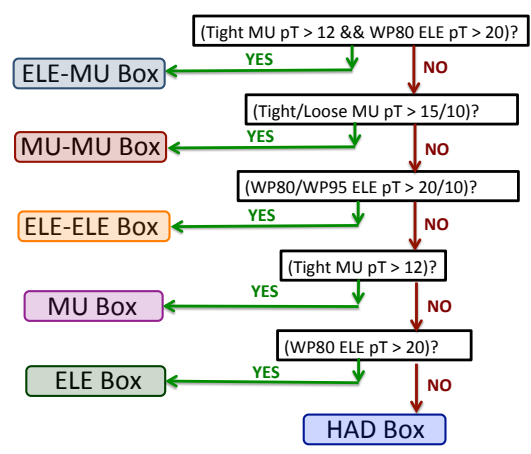

Figure 1: Flow diagram of box classification logic. The box selection proceeds according to a box hierarchy in order to ensure complete orthogonality of box selections and to resolve ambiguities when an event satisfies more than one box's selection criteria.
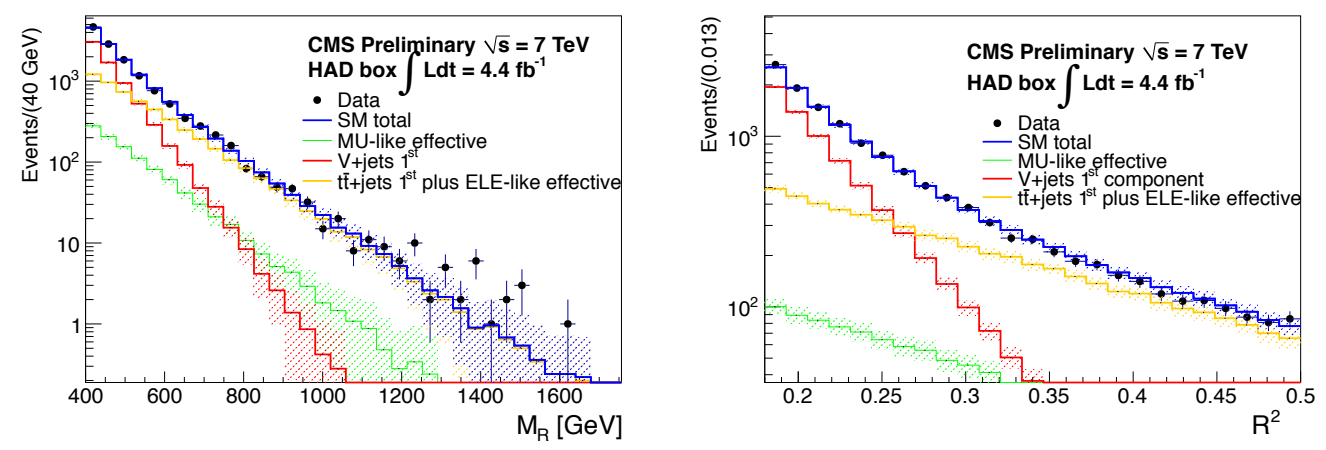

Figure 2: Projection of the 2D fit result on $M_{R}$ (left) and $R^{2}$ (right) for the hadronic box in the inclusive razor analysis of the CMS $20117 \mathrm{TeV}$ proton-proton dataset. The blue histogram is the total SM prediction as obtained from a single pseudo-experiment based on the 2D fit. The green, red, and yellow histograms show the breakdown of the SM prediction into separate components as returned by the fit. The fit is performed in the $R^{2}-M_{R}$ sideband and projected into the signal region. Only the statistical error on the total SM background prediction is shown in these projections.

\section{The Razor-b and Razor- $\tau$ Analyses}

The razor inclusive analysis is mainly sensitive to models featuring first and second generation quarks and leptons in their final state. In order to gain sensitivity to models in which the third generation dominates, the inclusive analysis was repeated on a subset of the data that featured at least one b-tagged jet [5] or at least one identified tau lepton [6] and interpreted in relevant simplified models (SMS). In SMS, introduced in Refs. [7], a limited set of hypothetical particles and decay chains are defined to describe a given topological signature. Specific applications of these ideas have appeared in Refs. [8]. Limits on direct stop and sbottom production from the Razor-b analysis are shown in Fig. 4, while many further results can be found in Ref. [9]. 


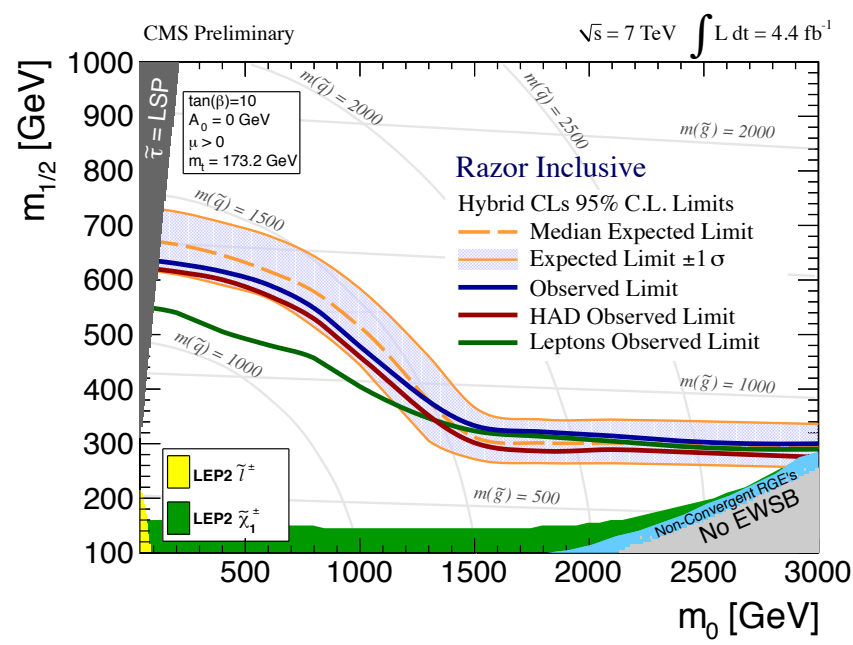

Figure 3: Observed (solid blue curve) and median expected (dot-dashed curve) 95\% CL limits in the $\left(m_{0}\right.$, $m_{1 / 2}$ ) CMSSM plane with $\tan \beta=10, A_{0}=0, \operatorname{sign}(\mu)=+1$ from the razor inclusive analysis. The \pm one standard deviation equivalent variations in the uncertainties are shown as a band around the median expected limit. Shown separately the observed hadronic-only (solid crimson) and leptonic-only (solid green) $95 \% \mathrm{CL}$ limits.
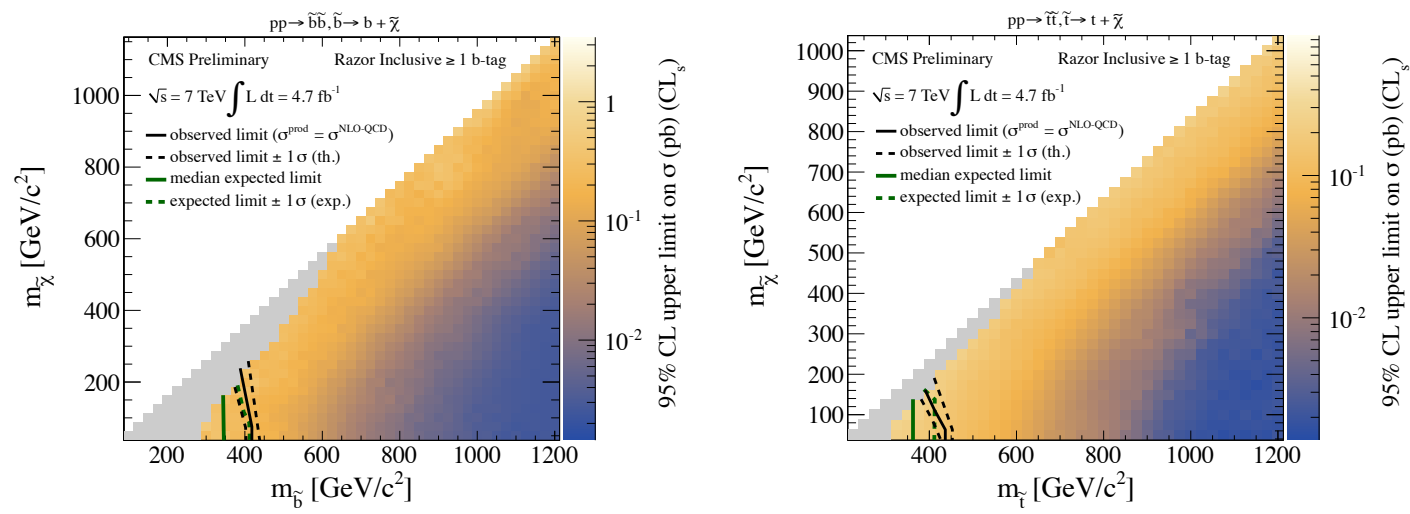

Figure 4: Exclusion cross-section vs. model spectrum T2bb (right) and T2tt (right) for the Razor-b analysis. Color-scale (z-axis) indicates the observed cross-section upper limit for each model point, as a function of the mass of the produced particle and the LSP mass. Solid black line indicates observed exclusion region, assuming nominal NLO+NLL SUSY production cross section. The solid grey region indicates model points where the analysis was found to have dependence on initial state radiation modelling in the simulation of signal events above a pre-defined tolerance; no interpretation is presented for these model points. 


\section{The Razor Multijet Analysis}

The inclusive razor analysis is particularly sensitive to models that produce fewer jets in the final state and feature sizeable transverse missing energy. However, some important areas of the new physics phase space fall outside of the acceptance, due to e.g. the intrinsic transverse missing energy resolution in the HLT. This is particularly important in searches for direct stop production for the decay mode in which both top quarks decay hadronically, which has the largest branching fraction. In this case, most signal events have small values of $R^{2}$, and are not selected by the razor triggers. To probe this final state directly, a dedicated analysis [10] was performed that made use of the CMS multijet triggers, which require 4,6 , or 8 calorimeter jets but do not use the missing transverse energy vector. Thus, the low- $R^{2}$ region of phase space can be probed for events with multiple jets. To give maximum sensitivity to direct stop production while reducing the major backgrounds considerably, events were required to have 6 offline jets, at least one of which was identified as a b-jet, and no identified leptons. The methodology introduced in Sec. 3 was then used to extract the shape of the SM background in a control region and then to extrapolate out into the signal region. No significant discrepancy was seen between the predicted and observed yields, and so limits were again set in a number of relevant SMS. Fig. 5 shows the expected and observed limits for direct stop production, fermionic $t^{\prime}$ pair-production, and for gluino pair production where the gluinos decay $\tilde{g} \rightarrow t t \tilde{\chi}_{0}$. The expected limits are strong, however, the observed limits are considerably lower due to a signal-like fluctuation of the background.
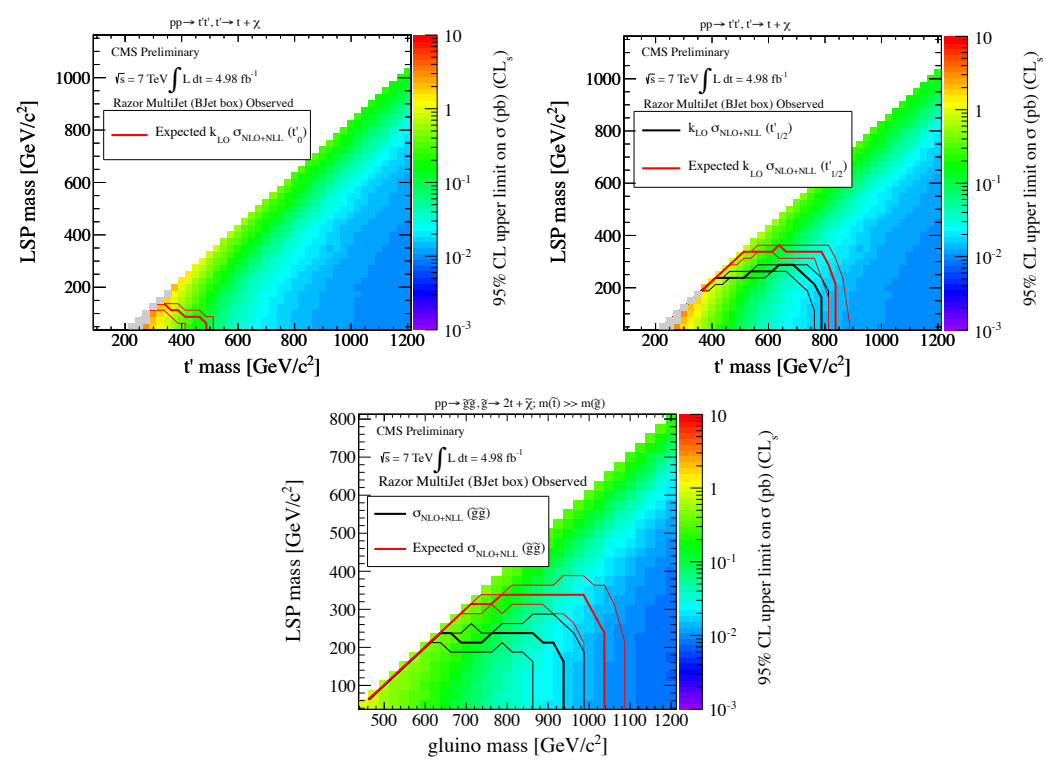

Figure 5: Expected and observed 95\% CL limits for direct-stop production (top left), fermionic $t^{\prime}$ pair production (top right), and gluino pair production (bottom). The theoretical uncertainties arising from scale and parton distribution function uncertainties are also shown by the narrow lines. The colour scale shows the model independent cross-section excluded in this SMS, while the solid grey region is explained in the caption of Fig. 4. 


\section{Conclusion}

The razor variables provide an excellent way to search for the production of heavy new states, which then decay into both visible and invisible particles. A complex methodology, based on estimating the shape of the SM background and then using this to extrapolate into multiple signal regions, has been developed and used in a number of different final states, including those featuring few jets or many, and with or without third-generation particles. While no discovery has been made in the $7 \mathrm{TeV}$ data, stringent limits have been set in a number of important benchmark models, including the CMSSM and SMS describing the pair production of stops, sbottoms, and gluinos.

\section{References}

[1] CMS Collaboration, R. Adolphi et. al., The CMS experiment at the CERN LHC, JINST 3 (2008) S08004.

[2] C. Rogan, Kinematics for new dynamics at the LHC, 1006.2727.

[3] CMS Collaboration, S. Chatrchyan et. al., Inclusive search for squarks and gluinos in pp collisions at sqrt $(s)=7 \mathrm{TeV}, 1107.1279$.

[4] CMS Collaboration, S. Chatrchyan et. al., Search for supersymmetry with the razor variables at CMS, CMS PAS SUS-12-005 (2012).

[5] CMS Collaboration, S. Chatrchyan et. al., Search for supersymmetry in final states with $b$-jets using the razor variables at sqrt( $(s)=7 \mathrm{TeV}$, CMS PAS SUS-11-024 (2012).

[6] CMS Collaboration, S. Chatrchyan et. al., Search for supersymmetry with taus using the razor variables, CMS PAS SUS-11-029 (2012).

[7] B. Knuteson and S. Mrenna, BARD: Interpreting new frontier energy collider physics, hep-ph / $6602101 ;$ N. Arkani-Hamed et. al., MARMOSET: The Path from LHC Data to the New Standard Model via On-Shell Effective Theories, hep-ph/0703088.

[8] J. Alwall, P. Schuster and N. Toro, Simplified Models for a First Characterization of New Physics at the LHC, Phys. Rev. D79 (2009) 075020 [0 810 . 3921]; J. Alwall, M.-P. Le, M. Lisanti and J. G. Wacker, Model-Independent Jets plus Missing Energy Searches, Phys.Rev. D79 (2009) 015005 [0809.3264]; D. Alves et. al., Simplified Models for LHC New Physics Searches, 1105.2838 ; D. S. Alves, E. Izaguirre and J. G. Wacker, Where the Sidewalk Ends: Jets and Missing Energy Search Strategies for the 7 TeV LHC, JHEP 1110 (2011) 012 [1102 . 5338]; M. Papucci, J. T. Ruderman and A. Weiler, Natural SUSY Endures, 1110.6926.

[9] CMS Collaboration, S. Chatrchyan et. al., Interpretation of searches for supersymmetry, CMS PAS SUS-11-016 (2012).

[10] CMS Collaboration, S. Chatrchyan et. al., A search for the decays of a new heavy particle in multijet events with the razor variables at CMS in pp collisions at sqrt(s) $=7 \mathrm{TeV}, C M S$ PAS SUS-12-009 (2012). 